\title{
Controlled Triangularization Fingerprint Verification Inscribed in a Rhombus Using Fuzzy Feature Matching
}

\author{
R. Kavitha Jaba Malar and V. Joseph Raj
}

\begin{abstract}
This paper proposes a novel method, a Fuzzy Feature Match (FFM) based on a controlled triangle feature set inscribed in a rhombus to match the deformed fingerprints. The fingerprint is represented by the fuzzy feature set. The fuzzy features set similarity is used to analyze the similarity among fingerprints. A fuzzy similarity for two triangles is introduced and then a similarity vector including the triangle-level similarities for all triangles and the rhombus in two fingerprints are constructed. Accordingly, a similarity vector pair is defined to illustrate the similarities between two fingerprints. The FFM method shows the similarity vector pair to a normalized value which quantifies the overall image to image similarity. The algorithm has been evaluated with Noorul Islam (NI) university staff members' fingerprint database. Experimental results confirm that the proposed FFM based on the local triangle feature set inscribed in a rhombus is a reliable and effective algorithm for fingerprint matching with nonlinear distortions.
\end{abstract}

Index Terms - Controlled triangularization, distortion, fuzzy features, minutia.

\section{INTRODUCTION}

Fingerprint recognition has been applied to identify criminals and currently it is being increasingly used for personal identification in a day-to-day life. Various fingerprint recognition techniques on fingerprint acquisition, enhancement, matching and classification are developed and advanced rapidly. However, there are still difficult and challenging tasks in this field. The main difficulty in matching two fingerprint impressions of the same finger is to deal with the nonlinear distortions, which are caused by the acquisition process. The performance of automatic fingerprint verification algorithms depends on Local ridge characteristics because ridge direction and minutiae such as ridge bifurcations are used for matching as shown in Fig.1.

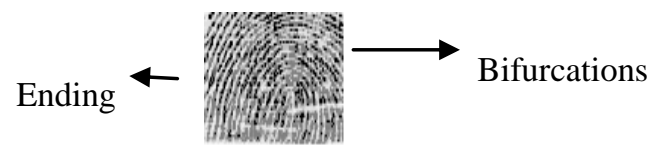

Fig. 1. Ridge structure of a fingerprint.

The ridges can be easily detected and minutiae can be correctly extracted from a thinned image on an ideal fingerprint image. The quality of many fingerprints is often poor due to the skin injuries and the surfaces in which they were taken. A critical step in studying the statistics of

Manuscript received April 9, 2013; revised June 10, 2013.

R. Kavitha Jaba Malar is with Mother Teresa Women's university, Kodaikanal, India. (email: kavith_in2000@yahoo.co.in).

V. Joseph Raj is with the Department of Computer Science, Kamaraj College, Manonmaniam Sundaranar University, Thoothukudi, India. fingerprint minutiae is to reliably extract minutiae from fingerprint images. So, there is a need to employ image enhancement techniques prior to minutiae extraction for obtaining a more reliable estimate of Minutiae locations. Some methods measured the forces and torques on the scanner directly with the aid of specialized hardware, if excessive force is applied or the estimated distortion is too large, the captured fingerprint image will be deleted. However, these methods do not work with the collected images. A FFM based on the local triangular feature set inscribed in a rhombus is proposed. It is a reliable and effective algorithm for fingerprint matching with nonlinear distortions.

\section{LITERATURE SURVEY}

In some methods, to match the deformed fingerprints a bounding box was used. A fixed-sized bounding box was used during the matching process in Jain's algorithm [1]. Luo et al. [2] improved this algorithm, a changeable bounding box had been applied during the matching process which is more robust in nonlinear deformations between the images. By using distance normalization and local alignment to deal with the nonlinear distortion Lee et al. [3] addressed minutiae-based fingerprints matching algorithm. Ratha et al. [4] used the local neighborhood structures to get the corresponding Minutiae pairs. Maio [5] proposed a plastic distortion model to cope with the nonlinear deformations. The physical cause of the distortion is modeled by distinguishing three distinct concentric regions in a fingerprint. The model provides an accurate description of the plastic distortions, but it is difficult to accurately estimate the distortion parameters. Kovacs-Vajna [6] proposed a triangular matching method to cope with the strong deformation of fingerprint images. Chen et al. [7] proposed an algorithm based on fuzzy theory to deal with the nonlinear distortion. The method based on fuzzy theory, normalized fuzzy similarity measure was introduced to compute the similarity between the template and input fingerprints, Apart from the above mentioned methods, we studied a better way, Fuzzy Feature Match based on a local triangle feature set to match the deformed fingerprints [8] The fingerprint was represented by the fuzzy feature set: local triangle feature set. The similarity between the fuzzy feature set was used to characterize the similarity between fingerprints. A fuzzy similarity measure for two triangles was first introduced. The result was then extended to construct a similarity vector which includes the triangle-level similarities for all triangles in two fingerprints. A similarity vector pair was defined to illustrate the similarities between two fingerprints. The FFM measure mapped a similarity vector pair to a normalized quantity within the real interval $[0,1]$, 
which quantifies the overall image to image similarity. The algorithm was experimented and evaluated with the fingerprint database of NI University staff members [9]. Experimental results indicate that this algorithm works well with the nonlinear distortions. The authors of this paper proposed FFM based on the local triangular feature set inscribed in a rhombus and reported its efficiency.

\section{Process of Extracting Minutiae}

The distance between two Minutiae is generally greater than a threshold value. While near the spurious Minutiae, there are usually other spurious Minutiae, and they are often detected at the border of the fingerprint image.

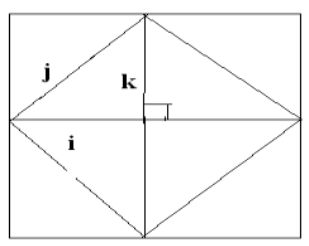

Fig. 2. Controlled triangle structure of the fingerprint.

The ridges can be easily detected and minutiae can be correctly extracted from a thinned image. The ridge structures in the poor-quality fingerprint images are not well defined and Minutiae cannot be correctly detected. They may be degraded and corrupted with elements of noise due to many factors including variations in skin and impression conditions. There are many approaches to construct triangles in the triangularization method, but there may be any number of triangles in a fingerprint. In the proposed method, we constructed a rhombus inside a fingerprint. It reduces the complexity.

\section{Controlled Triangle Feature SeT}

The first step is to define a controlled triangular feature set inscribed in a rhombus. The feature vector of a local triangle structure is defined by the distance between Minutiae, $F R T_{k}=$ $\left\{i, j, k, \Psi_{i}, \Psi_{j}, \Psi_{k}, A T, P T, A R, P R, I R R\right\}$, where $i, j, \mathrm{k}$ are the three minutiae, $A T$ and $P T$ are the area and the perimeter of the triangle and $A R, P R, I R R$ are the area, perimeter and inscribed radius of the rhombus respectively. The Rhombus is constructed by the triplets of Minutiae satisfying the following constraints; it means that a minutia must touch the edge of line drawn. The line must cover the four sides then the skeleton may be a square or a rectangle. Then construct a rhombus using the midpoints. There will be four triangles in a rhombus, which consists of feature vectors of all local triangles detected from a fingerprint. The rhombus is used to represent the image. The fingerprint matching is to find a similarity between two feature vector sets, one from the template and another from input fingerprint.

\section{A. Genuine Distorted Pattern Parameters Space}

Before measuring the similarity between the fuzzy feature set, we define the genuine distorted pattern parameter space. Suppose $F R T_{T k}=\left\{i, j, k, \Psi_{i}, \Psi_{j}, \Psi_{k}, A T, P T, A R, P R, I R R\right\}$ is a controlled local triangle feature in a template fingerprint and
$F R T_{I K}=\left\{i^{l}, j^{l}, k^{l}, \Psi_{i}^{l}, \Psi_{j}^{l}, \Psi_{k}^{l}, A T^{l}, P T^{l}, A R^{l}, P R^{l}, I R R^{l}\right\}$ is a controlled triangle feature in an input fingerprint, four distorted pattern parameters, vectors $l e n_{\text {diff, }}, \Psi_{\text {diff, }}, A T_{\text {diff }}$ and $P T_{\text {diff }}$ are calculated to construct the deformed pattern feature vector. To learn the genuine distorted pattern parameter, we applied a set of distorted fingerprint images to derive a genuine distorted pattern parameter space. We choose the corresponding training samples for each database. In the NI University staff members fingerprint databases, we used training set to derive the genuine distorted pattern parameter space. The database set contains fingerprint images captured from staff members' fingers, eight images for each finger. The image was acquired through the "Cross Match V300" optical sensor. The size of the image is $640 \times 480$ pixels with the resolution being about $500 \mathrm{dpi}$. In order to compute the genuine distorted pattern parameter space of the fingerprints, we matched those impressions from the same finger and trained the distorted pattern parameters.

\section{B. Fuzzy Feature Matching}

The similarity between the fuzzy features is used to characterize the similarity between fingerprints. We introduce a fuzzy similarity measure for four triangles inscribed in a rhombus and extend it to construct a similarity vector including the triangle-level similarity for all triangles in two fingerprints. The FFM method maps a similarity vector pair to a normalized quantity, within the interval $[0,1]$, which quantifies the overall image to image similarity.

\section{Fuzzy Similarity of Triangles}

The elements in the pattern parameter space construct the fuzzy feature set $\mathrm{G}$. The $V(G)$ denotes the volume of $\mathrm{G}$. The center of the fuzzy feature set $\mathrm{g}$ is used to represent feature set, which is defined as

$$
g=\frac{\sum_{\bar{f} \in G^{\bar{f}}}}{V(G)}
$$

We first, calculate the deformed pattern feature vector $f_{k}$ $\left(l e n_{\text {diff, }} \Psi_{\text {diff, }} O Z_{\text {diff, }}, \boldsymbol{\alpha}_{\text {diff }}\right)$. Then the degree of membership of the fuzzy feature set is measured. The modified Cauchy function is chosen due to its good expressiveness and high-computational efficiency. The membership function of the fuzzy feature set is defined as

$$
C(\bar{f})=\left\{\begin{array}{c}
1, \frac{1}{1+\left(\frac{\bar{f}-\bar{g}}{m}\right)^{a}} \\
\text { if } h(\bar{f}, \bar{g})=\text { True, otherwise }
\end{array}\right\}
$$

\section{Fuzzy Similarity between Fingerprints}

It is clear that the image-level similarity is constructed from triangle-level similarities. The FFM method is applied to provide an image to image similarity. The overall image to image similarity is calculated by summation of all the weighted entries of similarity vectors. The FFM method computes the inner products of similarity vectors with weight vectors. There are many options to choose weight vectors. We can take the location of the triangles into account and assign higher weights to triangles closer to the center of the 
fingerprint. Another option is the area scheme. It takes the area covered by the triangle as the weight based on the viewpoint that the triangle of the proper area in a fingerprint. In the FFM method, both area and center-favored schemes are used. Consequently, the FFM measure for template and input fingerprints is defined as

$$
\operatorname{Sim}=(1-p) w_{A}+w_{B} \quad L^{(T, I)}
$$

Here $w_{A}$ is the normalized area percentage of both template and input fingerprints, $w_{B}$ is the normalized weight which favours triangle near the image center, $p €[0,1]$ adjusts the significance of $w_{A}$ and $w_{B}$ and $L^{(T, I)}$ is the weighted entries of similarity vector of the overall image. In the paper the fingerprint is represented by the local triangle feature set inscribed in a rhombus. The similarity between template and input fingerprints is constructed by triangle similarities. It is difficult to get the closed-form equation. The minutiae number of the template and input fingerprints, respectively, is the probability that the similarity between the template and input fingerprints is greater than threshold. Here, we analyze the matched number of triplets of Minutiae which satisfy the entire criterion in the matching process, and the probability of the local triangle feature set matching model.

\section{EXPERIMENTAL RESULTS}

The proposed algorithm has been evaluated on the fingerprints of NI University staff members' database. This algorithm is compared with the methods described earlier. The proposed algorithm considerably reduces the complexity of computation. In the algorithm controlled four triangles inscribed in a rhombus is used for a finger. A better performance of $95 \%$ accuracy is also obtained. In the FFM using local triangularization method, infinite number of triangles is constructed for a fingerprint. In the new FFM using controlled triangularization inscribed in a rhombus, only four triangles are constructed for a fingerprint. The computation complexity in FFM based controlled triangularization method is more less than the local triangularization method.

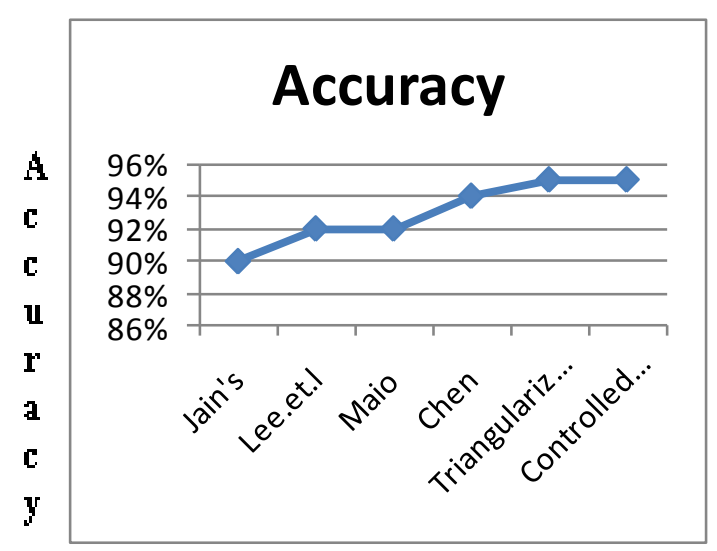

Fig. 3. Comparison of controlled triangularization method with conventional methods.

For finger1, $G=\{57,57,57,57\}$

$V(G)=\{13,13,13,13\}$

For finger2, $G=\{59,59,59,59\}$

$V(G)=\{15.4,15.4,15.4\}$
The performance of the feature vector is shown below (sample) in Table I, Table II, Table III and Table IV.

TABLE I: FEATURE VECTORS OF TRIANGLES IN FINGERPRINTS

\begin{tabular}{rcccccc}
\hline \hline Finger & $\boldsymbol{I}$ & $\boldsymbol{j}$ & $\boldsymbol{K}$ & $\boldsymbol{X}_{\boldsymbol{i}}$ & $\boldsymbol{X}_{\boldsymbol{j}}$ & $\boldsymbol{X}_{\boldsymbol{k}}$ \\
\hline $\mathbf{1 - 1}$ & 1.4 & 2.4 & 1.9 & $52^{0} 20^{1}$ & $37^{0} 81^{1}$ & $90^{0}$ \\
$\mathbf{1 - 2}$ & 1.4 & 1.9 & 2.4 & 90 & $35^{0} 41^{1}$ & $54^{0} 59^{1}$ \\
$\mathbf{1 - 3}$ & 2.4 & 1.9 & 1.4 & $35^{0} 41^{1}$ & $90^{0}$ & $54^{0} 59^{1}$ \\
$\mathbf{1 - 4}$ & 2.4 & 1.9 & 1.4 & $35^{0} 41_{1}$ & $90^{0}$ & $54^{0} 59^{1}$ \\
\hline \hline
\end{tabular}

TABLE II: FEATURE VECTORS OF THE RHOMBUS IN FINGERPRINTS $\mathrm{AR}=5.4 ; \mathrm{PR}=0.08 ; \mathrm{RR}=1.13$

\begin{tabular}{lllllcccc} 
Finger & $\boldsymbol{i}$ & $\boldsymbol{j}$ & $\boldsymbol{k}$ & $\boldsymbol{X}_{\boldsymbol{i}}$ & $\boldsymbol{X}_{\boldsymbol{j}}$ & $\boldsymbol{X}_{\boldsymbol{k}}$ & $\boldsymbol{A T}$ & $\boldsymbol{P T}$ \\
\hline $\mathbf{1 - 1}$ & 1.4 & 2.3 & 1.8 & $90^{0}$ & $51^{0} 30^{1}$ & $38^{0} 70^{1}$ & 1.26 & 2.52 \\
$\mathbf{1 - 2}$ & 1.4 & 2.3 & 1.8 & $90^{0}$ & $51^{0} 30^{1}$ & $38^{0} 70^{1}$ & 1.26 & 2.52 \\
$\mathbf{1 - 3}$ & 1.4 & 2.3 & 1.8 & $90^{0}$ & $51^{0} 30^{1}$ & $38^{0} 70^{1}$ & 1.26 & 2.52 \\
$\mathbf{1 - 4}$ & 1.4 & 2.3 & 1.8 & $90^{0}$ & $51^{0} 30^{1}$ & $38^{0} 70^{1}$ & 1.26 & 2.52 \\
\hline \hline
\end{tabular}

TABLE III: FEATURE VECTORS OF THE INNER RADIUS OF RHOMBUS IN

\begin{tabular}{|c|c|c|c|c|c|c|c|c|}
\hline \multicolumn{9}{|c|}{ FINGERPRINTS } \\
\hline Finger & $\bar{i}$ & $\bar{j}$ & $\bar{k}$ & $\overline{\bar{X}}$ & $\overline{X_{j}}$ & $\overline{X_{k}}$ & $A T$ & $P T$ \\
\hline T1 & 1.4 & 2.4 & 1.9 & 90 & $52^{0} 20^{1}$ & $37^{0} 80^{1}$ & 1.33 & 2.66 \\
\hline $\mathbf{T} 2$ & 1.4 & 2.4 & 1.9 & 90 & $52^{0} 20^{1}$ & $37^{0} 80^{1}$ & 1.33 & 2.66 \\
\hline T3 & 1.4 & 2.4 & 1.9 & 90 & $52^{0} 20^{1}$ & $37^{0} 80^{1}$ & 1.33 & 2.66 \\
\hline T4 & 1.4 & 2.4 & 1.9 & 90 & $52^{0} 20^{1}$ & $37^{0} 80^{1}$ & 1.33 & 2.66 \\
\hline
\end{tabular}

TABLE IV: FEATURE VECTORS SET PATTERN PARAMETERS

\begin{tabular}{ccccccccc}
\hline \hline $\boldsymbol{i}$ & $\boldsymbol{j}$ & $\boldsymbol{k}$ & $\boldsymbol{X}_{\boldsymbol{i}}$ & $\boldsymbol{X}_{\boldsymbol{j}}$ & $\boldsymbol{X}_{\boldsymbol{k}}$ & $\boldsymbol{A T}$ & $\boldsymbol{P T}$ & $\boldsymbol{I r R}$ \\
\hline $\mathbf{1 . 4}$ & 2.3 & 1.8 & $90^{0}$ & $51^{0} 30^{1}$ & $38^{0} 70^{1}$ & 1.26 & 2.52 & 5.04 \\
$\mathbf{1 . 4}$ & 2.3 & 1.8 & $90^{0}$ & $51^{0} 30^{1}$ & $38^{0} 70^{1}$ & 1.26 & 2.52 & 1.13 \\
\hline \hline
\end{tabular}

$\mathrm{IrR}=1.13 ; \mathrm{AR}=5.32 ; \mathrm{PR}=0.64$

\section{CONCLUSION}

This paper proposes a new method for deformed fingerprints matching. The fingerprint of controlled triangularization inscribed in a rhombus feature is represented by the fuzzy feature. These features are used to characterize the similarity between fingerprints. We introduce a fuzzy similarity measurement for two triangles and extend it to construct a similarity vector including the triangle-level similarity in two fingerprints. The similarity vector pair is defined to illustrate the resemblance between two fingerprints. The FFM method maps a similarity vector pair to a normalized quantity. The proposed algorithm has been evaluated with fingerprints of NI University staff members' database. Experimental results confirm that our algorithm works well with the nonlinear distortions. The proposed system reduces the complexity of the local triangularization method and produces better accuracy in matching. 


\section{REFERENCES}

[1] A. K. Jain, L. Hong and R. Bolle, "On-line fingerprint verification," IEEE Transactions on Pattern Analysis and Machine Intelligence, vol. 19, no.4, pp.302-313, Apr, 1997.

[2] X. P. Luo, J. Tian, and Y.Wu, "A minutia matching algorithm in fingerprint verification," in Proc. $15^{\text {th }}$ ICPR, sep. 2000, vol. 4, PP. 833-836.

[3] D. Lee, K. Choi and J. Kim, "A robust fingerprint matching algorithm using local alignment," in Proc. 16 ${ }^{\text {th }}$ ICPR, Aug. 2002, vol.3, pp.803-806.

[4] N. K. Ratha and R.M.Bolle, "Effect of controlled acquisition on fingerprint matching," in Proc. $14^{\text {th }} I C P R, 1998$, vol. 2, pp. 1659-1661.

[5] R. Cappelli, D. Maio and D. Maltoni, "Modeling plastic distortion in fingerprint images," in Proc. ICAPER, Mar.2001, pp.369-376.

[6] Z. M. Kovacs-Vajna, "A fingerprint verification system based on triangular matching and dynamic time warping," IEEE Transactions on Pattern Analysis and Machine Intelligence, vol.22, no. 11, pp. 1266-1276, Nov, 2000.

[7] H. Chen, J. Tian and X. Yang, "Fingerprint matching with registration pattern inspection," in Proc. Audio-and Video-Based Biometrie Person Authentication, 4th International Conference, Guildford, UK, 2003, pp. 327-334.

[8] X. J. Chen, J. Tian and X. Yang, "A new algorithm for distorted fingerprints matching based on normalized Fuzzy Similarity Measure," IEEE Trans. Image Process. vol. 15, no. 3, pp. 767-776, Mar.2006.

[9] R. Kavitha Jaba Malar and V. Joseph Raj, "Fingerprint verification using fuzzy feature matching, conference record," in Proc. International Conference on Emerging Trends in Engineering and Technology, Teerthanker Mahaveer University, 2012.

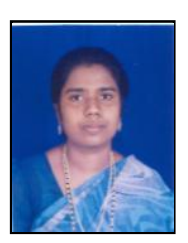

R. Kavitha Jaba Malar is from India and was born on July 29, 1976. She received the MCA degree from Manonmaniam Sundaranar University, Tirunelveli, India .on 2001. Where she is currently pursuing the $\mathrm{Ph}$.D. degree at Mother Teresa Women's university, Kodaikanal, India. She is presently working as a software developer at SRM InfoTech.

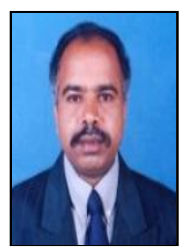

V. Joseph Raj received his $\mathrm{PhD}$ degree from Manonmaniam Sundaranar University, Tirunelveli, India and Post Graduate degree in Anna University, Chennai, India. He is presently working as a Professor and Head of the Department of Computer Science, Kamaraj College, Manonmaniam Sundaranar University, Thoothukudi, India. He has also worked as an Associate Professor in Computer Engineering in European University of Lefke, North Cyprus. He is serving as the Chairman of Computer Science Board of Studies of Manonmaniam Sundaranar University. He is guiding $\mathrm{PhD}$ scholars of various Indian universities. He has a vast teaching experience of about 25 years and research experience of about 18 years. His research interests include neural network, digital image processing, biometrics, network security and operations research. Research experience of about 18 years. His research interests include neural network, digital image processing, biometrics, network security and operations research. 